\title{
A Multi-Messenger Picture of Galaxy Mergers: Neutrinos and Electromagnetic Emissions
}

\author{
Chengchao Yuan ${ }^{* 1}$, Kohta Murase ${ }^{1,2}$ and Peter Mészáros ${ }^{1}$ \\ ${ }^{1}$ Department of Physics; Department of Astronomy \& Astrophysics; Center for Particle and \\ Gravitational Astrophysics, The Pennsylvania State University, University Park, PA 16802, \\ USA \\ ${ }^{2}$ Yukawa Institute for Theoretical Physics, Kyoto University, Kyoto, Kyoto 606-8502, Japan \\ E-mail: cxy52@psu.edu
}

\begin{abstract}
The merger of dark matter halos and the gaseous structures embedded in them, such as galaxies and clusters of galaxies, results in strong shocks. These shocks will produce high-energy neutrinos and gamma-rays through inelastic $p p$ collisions. In this work, we formulate the redshift dependence of the shock velocity, galactic radius, halo gas content and galactic/intergalactic magnetic fields over the dark matter halo distribution up to a redshift $z=10$. We find that highredshift mergers contribute a significant amount of the cosmic-ray (CR) luminosity density, and the resulting neutrino spectra could explain a large part of the observed diffuse neutrino flux above 0.1 PeV up to several PeV without violating the $\gamma$-ray constraint. Moreover, the secondary electrons/positrons from pion decays can produce observable emissions through synchrotron radiation and inverse Compton scattering while propagating in the host galaxies. We demonstrate that these emissions can explain the radio and X-ray fluxes of the merging galaxies such as NGC 660 and NGC 3256. In the future, with the improved sensitivity of the Cherenkov Telescope Array (CTA) and IceCube-Gen2, our model for the merging galaxies can be further tested by gamma-ray and neutrino observations.
\end{abstract}

36th International Cosmic Ray Conference -ICRC2019-

July 24th - August 1st, 2019

Madison, WI, U.S.A.

\footnotetext{
* Speaker.

${ }^{\dagger}$ This research was partially supported by NASA NNX13AH50G (C.C.Y., P.M.), and the Alfred P. Sloan Foundation and NSF grant PHY-1620777 (K.M.).
} 


\section{Introduction}

Since the first detection of high-energy (HE) astrophysical neutrinos by the IceCube Neutrino Observatory, a diffuse background of astrophysical neutrinos in the energy range from $\sim 10 \mathrm{TeV}$ to several $\mathrm{PeV}$ has been unveiled and measured to a higher precision [1-3]. The isotropic distribution of these neutrinos suggests that a large part of them come from extragalactic sources. Despite the physical nature of the sources of these diffuse neutrinos still remains in dispute, many candidates have been proposed and studied $[4,5]$. It is generally accepted that these neutrinos are generated by charged pion $\left(\pi^{ \pm}\right)$decays, and that these secondary pions are products of the CR particles undergoing hadronuclear $(p p)$ or photohadronic $(p \gamma)$ interactions between the CRs and ambient gas content or photon field. Not only HE neutrinos, these interactions can also generate neutral pions $\left(\pi^{0}\right)$ as well, which subsequently decay into pairs of $\gamma$-ray photons. Hence, the multi-messenger consideration of the cumulative neutrino and $\gamma$-ray backgrounds is helpful and necessary to constrain the origin of these HE astrophysical neutrinos.

In this work, we focus on the halo and galaxy mergers as the origin of HE neutrinos. The motivation is that when dark matter halos merge, the galaxies in these halos also merge, and the collision of the cold gas in the merging galaxies leads to shocks on a galactic scale in the galactic interstellar medium (ISM). The subsequent $p p$ collisions between the shock-accelerated CRs and the thermal atomic nuclei in the gaseous environment are the major mechanism that generates HE neutrinos and $\gamma$ rays in these systems. We consider in $\$ 2$ this scenario of both galactic-scale shocks in the galactic ISM and group/cluster-scale shocks in the intergalactic medium (IGM) across redshifts, whereas [6] studied only major galaxy mergers (mergers of two galaxies of approximately the same size) at $z \sim 1$.

The pions produced in the $p p$ collisions between shock-accelerated CR ions and the galaxy gas generate, besides HE neutrinos and $\gamma$ rays, copious quantities of HE electron-positron pairs. These HE leptons may produce observable synchrotron emissions while propagating inside the galactic magnetic fields. In general, secondary electrons, rather than shock-accelerated primary electrons, are more natural to explain the electromagnetic emissions in merging galaxies, since the fraction of plasma energy that is directly deposited to electrons, is at least two orders lower than the proton acceleration efficiency, e.g. $K_{e / p}=\eta_{e} / \eta_{p} \lesssim 10^{-4}-10^{-2}$ [7,8]. The ratio of energies converted to the primary leptons (from shock accelerations) and to the secondary electrons and positrons is approximately

$$
\frac{\mathscr{E}_{e, \text { primary }}}{\mathscr{E}_{e, \mathrm{sec}}} \simeq \frac{6 \eta_{e}}{\min \left[1, f_{p p, \mathrm{~g}}\right] \eta_{p}} \lesssim 10^{-1},
$$

where $f_{p p, \mathrm{~g}}$ is the $p p$ optical depth in the emitting region. Therefore, in our model with the typical electron/proton acceleration efficiencies, emission from primary electrons directly accelerated in shocks is subdominant compared to that from secondary electrons and positrons from $p p$ collisions and pion decays.

In $\$ 3$ we formulate a one-zone model that is capable of explaining the radio and X-ray observations of specific systems using synchrotron and synchrotron self-Compton (SSC), or external inverse Compton (EIC), emissions from HE secondary electron-positron pairs. Here, the EIC is caused by scatterings with the cosmic microwave background (CMB), infrared/optical starlight (SL), and extragalactic background light (EBL). The detectability of galaxy mergers as a point 
source using future neutrino and $\gamma$-ray detectors, as well as a conclusive discussion, are presented in $\S 4$.

\section{Diffuse Neutrino and $\gamma$-ray Backgrounds}

Assuming that the CRs are mainly protons and the spectral index is $s=2$, the comoving CR energy input rate per the logarithm of the CR energy $\varepsilon_{p}$ can be obtained by integrating over the halo mass $M_{h}$

$$
\varepsilon_{p} Q_{\varepsilon_{p}}(z)=\frac{E_{\mathrm{merger}}}{t_{\mathrm{age}} \mathscr{C}}=\eta_{p} \mathscr{C}^{-1} \int_{M_{\min }}^{M_{\max }} d M_{h}\left[\frac{1}{2} \xi_{g}\left(M_{h}, z\right) M_{h} v_{s}^{2}\right] \frac{d N}{d M_{h}} \frac{P\left(M_{h}, z\right)}{t_{\mathrm{age}}},
$$

where $\xi_{g}\left(M_{h}, z\right)=M_{\text {gas }} / M_{h}$ is the mass fraction in gas form, $\eta_{p}$ is the fraction of shock energy that is deposited in CRs (nominally taken as 0.1$), v_{s}$ is the shock velocity and $\mathscr{C}=\ln \left(\varepsilon_{p}^{\max } / \varepsilon_{p}^{\min }\right)$ is the normalization factor for a CR spectrum $N\left(\varepsilon_{p}\right) \propto \varepsilon_{p}^{-2}$. In this equation, the halo gas fraction $\xi_{g}$ can be derived from the stellar mass function $M_{*}\left(M_{h}\right)$ [9] and the gas fraction in normal galaxies $f_{\mathrm{g}}=M_{\mathrm{gas}} /\left(M_{\mathrm{gas}}+M_{*}\right)[10]$, while $\frac{d N}{d M_{h}} \frac{P\left(M_{h}, z\right)}{t_{\text {age }}}$ plays the role of halo merger rate. After the CRs are accelerated in the shock, they will propagate in the host galaxy and cluster. In this process, neutrinos and $\gamma$-rays are generated from pions produced in inelastic $p p$ collisions. The meson production efficiency is $1-e^{-f_{p p}}$ where $f_{p p}=c \kappa_{p p} \sigma_{p p} g(z) \sum n_{i, 0} t_{i}$ is the effective $p p$ optical depth. In this expression, $n_{i, 0}$ represents the local gas density of the medium, for example, galaxies and clusters; $\sigma_{p p}$ is the $p p$ cross section; $\kappa_{p p} \simeq 0.5$ is the inelasticity coefficient; $t_{i}$ is the interacting time and $g(z)$ is the redshift evolution of the gas density. The $p p$ optical depth $f_{p p}$ should be parameterized separately for proton-proton collisions in galaxies and clusters [11], e.g. $f_{p p}^{\mathrm{g}}$ and $f_{p p}^{\mathrm{cl}}$, due to the distinct physical conditions.

Another thing we need to keep in mind is that halo mergers will also lead to galaxy cluster mergers, after some halos have grown above a certain size that may be taken approximately of the order $M_{h} \sim 10^{13} M_{\odot}$. We simplify the calculations as follows. For low-mass mergers, we expect that the $p p$ interactions occur mainly in gas with a galactic ISM density, while for high-mass mergers the $p p$ interactions occur mainly in IGM. In addition, there will be a subdominant component of $p p$ interactions due to CRs that escape from the colliding galaxy system into the IGM. Thus, we expect that the three-flavor neutrino production rate consists of a galaxy part $\varepsilon_{v} Q_{\varepsilon_{v}}^{(\mathrm{g})}$ and a cluster part $\varepsilon_{v} Q_{\varepsilon_{v}}^{(\mathrm{cl})}$ plus a weaker galaxy-cluster term:

$$
\begin{aligned}
\varepsilon_{v} Q_{\varepsilon_{v}}^{(\mathrm{g})} & =\frac{1}{2}\left(1-e^{-f_{p p}^{\mathrm{g}}}\right) \varepsilon_{p} Q_{\varepsilon_{p}}^{(\mathrm{LM})} \\
\varepsilon_{v} Q_{\varepsilon_{v}}^{(\mathrm{cl})} & =\frac{1}{2}\left[\left(1-e^{-f_{p p}^{\mathrm{cl}}}\right) \varepsilon_{p} Q_{\varepsilon_{p}}^{(\mathrm{HM})}+\eta\left(1-e^{-f_{p p}^{\mathrm{cl}}}\right) e^{-f_{p p}^{\mathrm{g}}} \varepsilon_{p} Q_{\varepsilon_{p}}^{(\mathrm{LM})}\right],
\end{aligned}
$$

where the energies of the neutrinos and CR protons are connected by $\varepsilon_{v} \simeq 0.05 \varepsilon_{p}$. In Eq. 2.2, $\varepsilon_{p} Q_{\varepsilon_{p}}^{(\mathrm{LM})}$ and $\varepsilon_{p} Q_{\varepsilon_{p}}^{(\mathrm{HM})}$ correspond to $\mathrm{CR}$ injections from the galaxy mergers in low-mass halos and cluster mergers in high-mass halos, respectively. For completeness, we introduce a parameter $\eta \lesssim 1$ to represent the fraction of galaxy mergers that occur inside clusters, which lead to some CRs escaping into the IGM. 


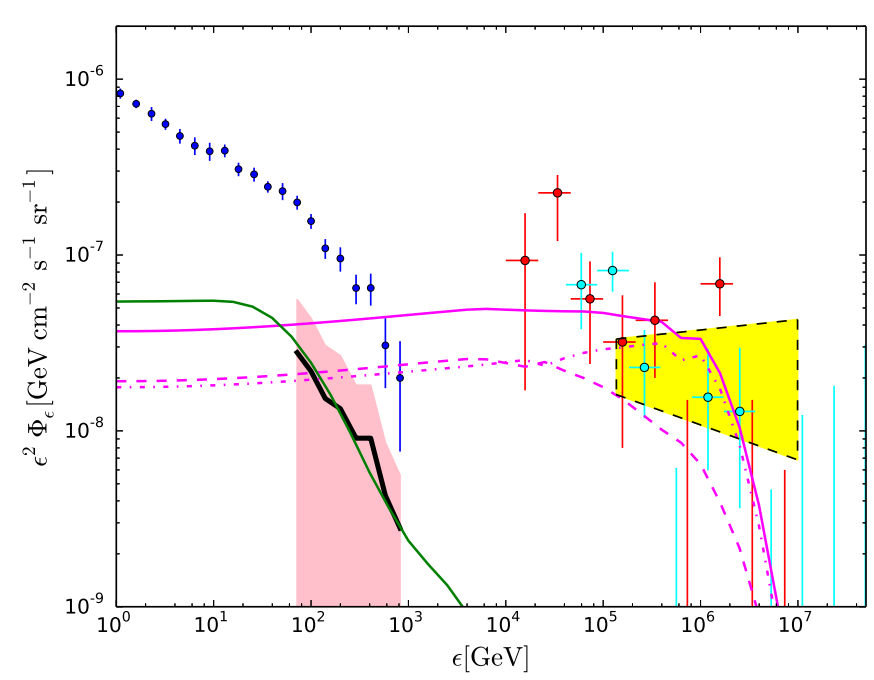

Figure 1: Three-flavor neutrino (magenta line) and $\gamma$-ray (green line) fluxes from halo mergers with the local galaxy radius $R_{\mathrm{g}, 0} \simeq 10 \mathrm{kpc}$ and the local shock velocity $v_{s, 0} \simeq 300 \mathrm{~km} \mathrm{~s}^{-1}$. Galaxy and cluster contributions to the overall neutrino flux are illustrated as the dashed and dash-dotted lines, respectively

Given the neutrino input rate, the all-flavor neutrino flux can be expressed as

$$
\varepsilon_{v}^{2} \Phi_{\varepsilon_{v}}=\frac{c}{4 \pi} \int \frac{\varepsilon_{v} Q_{\varepsilon_{v}}^{(\mathrm{g})}+\varepsilon_{v} Q_{\varepsilon_{v}}^{(\mathrm{cl})}}{(1+z)}\left|\frac{d t}{d z}\right| d z
$$

Considering the branch ratio between the charged and neutral pions from $p p$ collisions, the initial neutrino and $\gamma$-ray spectra are related by $\varepsilon_{\gamma}^{2} \Phi_{\varepsilon_{\gamma}}=\left.\frac{2}{3} \varepsilon_{v}^{2} \Phi_{\varepsilon_{v}}\right|_{\varepsilon_{v}=0.5 \varepsilon_{\gamma}}$. Different from neutrinos that can reach the earth without being absorbed, the HE $\gamma$ rays undergo $\gamma \gamma$ annihilations with low energy photon backgrounds including CMB and infrared EBL while propagating in the Universe. Hence, an attenuation factor $\exp \left(-\tau_{\gamma \gamma}\right)$ is required in the integration over redshift, where $\tau_{\gamma \gamma}$ is the $\gamma \gamma$ optical depth. The attenuated $\gamma$-ray flux is then

$$
\varepsilon_{\gamma}^{2} \Phi_{\varepsilon_{\gamma}}=\frac{c}{4 \pi} \int \frac{2}{3}\left[\frac{\varepsilon_{v} Q_{\varepsilon_{v}}^{(\mathrm{g})}+\varepsilon_{v} Q_{\varepsilon_{v}}^{(\mathrm{cl})}}{(1+z)}\left|\frac{d t}{d z}\right|\right] \times \exp \left[-\tau_{\gamma \gamma}\left(\varepsilon_{\gamma}, z\right)\right] d z
$$

with $\varepsilon_{p}=10 \varepsilon_{\gamma}(1+z)$. Moreover, the the electron-positron pairs produced in the $\gamma \gamma$ annihilations will subsequently initiate the electromagnetic cascade through inverse Compton scattering with low-energy photon backgrounds. In this paper, for simplicity, we use the universal form for the resulting cascaded $\gamma$-ray spectrum given by [12].

The diffuse neutrino and $\gamma$-ray fluxes are shown in Fig. 1, together with the IceCube-observed astrophysical neutrinos. The red points and cyan points correspond to the three-flavor averaged neutrino flux [2] and 6-year high-energy starting events (HESE; [13]), respectively. The Fermi-LAT observed total extragalactic $\gamma$-ray background (EGB) [14] is shown by the blue points. The yellow area is the best fit to the upcoming muon neutrinos scaled to three-flavor. From this figure, we find that the halo and galaxy mergers can broadly fit a significant fraction of the IceCube data without violating the nonblazar EGB (the pink area), since the high-redshift $(z \gtrsim 2-3)$ can contribute a significant portion to the total CR luminosity density. In this case, the $\gamma$ rays are sufficiently attenuated, which alleviates the tension between the neutrino and $\gamma$-ray backgrounds. 


\section{Secondary Radio and X-ray Emissions}

The hadronuclear scenario for the galaxy mergers also predicts a copious amount of HE electron-positron pairs in the merging region. These high-energy leptons may produce observable synchrotron emissions while propagating inside the galactic magnetic fields. Here, considering the conservation of lepton numbers, we approximate the total electron-positron injection spectrum to be similar with the neutrino production spectrum $(s=2)$

$$
\varepsilon^{2} \mathscr{N}_{e}(\varepsilon)=\frac{1}{3} \varepsilon^{2} \frac{d N_{v}}{d \varepsilon}=\frac{1}{12} \eta_{p} \mathscr{C}^{-1} M_{\mathrm{g}} v_{\mathrm{s}}^{2} \times \min \left[1, f_{p p, \mathrm{~g}}\right]_{\varepsilon_{p} \simeq 20 \varepsilon}
$$

where $M_{\mathrm{g}}$ is the gas mass of the merging region and the other symbols have the same meaning with Eq. 2.1. As galaxies merge, strong shocks occur with a complicated morphology over a galaxy scale, while merging cores of the two galaxies lead to a dense core region. Particles are accelerated by the shocks, and then will be distributed in a galaxy scale. The CRs diffusing in the core region will make neutrinos and gamma-rays efficiently. In this work, as a simplified approximation without covering the details of the shock structure, we assume that shocks are CR accelerators that inject high-energy CRs into the core region of the merging systems and initiate subsequent interactions. After leaving the accelerator, the particles can propagate diffusively or get advected away through galactic winds; therefore, the net escape rate is the sum of diffusion rate and advection rate, e.g., $t_{\mathrm{esc}}^{-1} \approx t_{\mathrm{diff}}^{-1}+t_{\mathrm{ad}}^{-1}$. In this work, to get the electron distribution inside the galaxy, we solve the transport equation of a simplified leaky-box model

$$
\frac{\partial N_{e}}{\partial t}=Q(\varepsilon, t)-\frac{N_{e}}{t_{\mathrm{esc}}}+\frac{\partial}{\partial \varepsilon}\left[b(\varepsilon) N_{e}(\varepsilon, t)\right]
$$

where $b(\varepsilon)$ is the electron energy loss rate due to synchrotron radiation, SSC/EIC and advection $\left(b_{\mathrm{ad}} \simeq \varepsilon / t_{\mathrm{ad}}\right)$. In this isotropic transport equation, the source function $Q(\varepsilon, t)$ is the differential injection rate of electrons/positrons and can be explicitly written as

$$
Q(\varepsilon, t)=\frac{\mathscr{N}_{e}(\varepsilon) \chi(\varepsilon)}{t_{\mathrm{dyn}}} \times \min \left\{1, e^{-\frac{t-t_{\mathrm{dyn}}}{\text { tesc }}}\right\},
$$

where $N_{e}(\varepsilon)$ represents the cumulative injection spectrum for all secondary particles produced during the merger, $t_{\mathrm{dyn}} \simeq R_{\mathrm{g}} / v_{s}$ is the dynamic time, the modification factor $\chi(\varepsilon)=1+\exp \left(-\varepsilon_{\gamma \gamma}^{\mathrm{cut}} / 2 \varepsilon\right)$ includes the additional injections via two-photon annihilation, $\gamma \gamma \rightarrow e^{-} e^{+}$, considering that the core region can be opaque to high-energy gamma-ray photons above a certain threshold energy $\varepsilon_{\gamma \gamma}^{\mathrm{cut}}$.

With the above, we are able to calculate the synchrotron and SSC/EIC fluxes. In this work, we present an application to the interacting system NGC 660 and show that our model can be used to explain the radio and X-ray observations. NGC 660 is usually believed as a galaxy formed by the collision and merger of two galaxies. The distance to us is $d_{L} \sim 12.3 \mathrm{Mpc}$. [15] showed that the deconvoluted angular size of the radio and X-ray emitting region is less than $10 \operatorname{arcsec}$ or equivalently the radius $R_{\mathrm{g}} \simeq 0.5 \mathrm{kpc}$. Hence, in our calculations, we use $0.5 \mathrm{kpc}$ as the fiducial radius of the core region. In addition, polarization studies demonstrate that the average magnetic field of the merging region is $B \simeq 16 \pm 5 \mu G$ [16]. Using these numbers as fiducial parameters as well as the radio and X-ray observations ${ }^{1}$, Fig. 2 illustrates the radio and X-ray constraints on

\footnotetext{
${ }^{1}$ A full list of references can be found in the page NED:INDEX NGC 660
} 

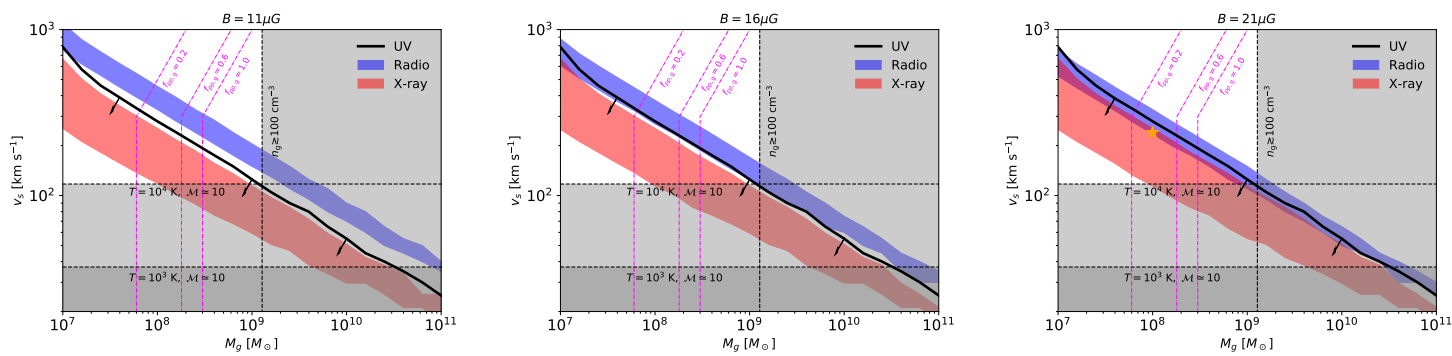

Figure 2: Constraints on $M_{\mathrm{g}}-v_{s}$ plane from radio, UV and X-ray tolerance areas. From left to right, magnetic fields are assumed to be $B=11 \mu \mathrm{G}, 16 \mu \mathrm{G}$ and $21 \mu \mathrm{G}$.
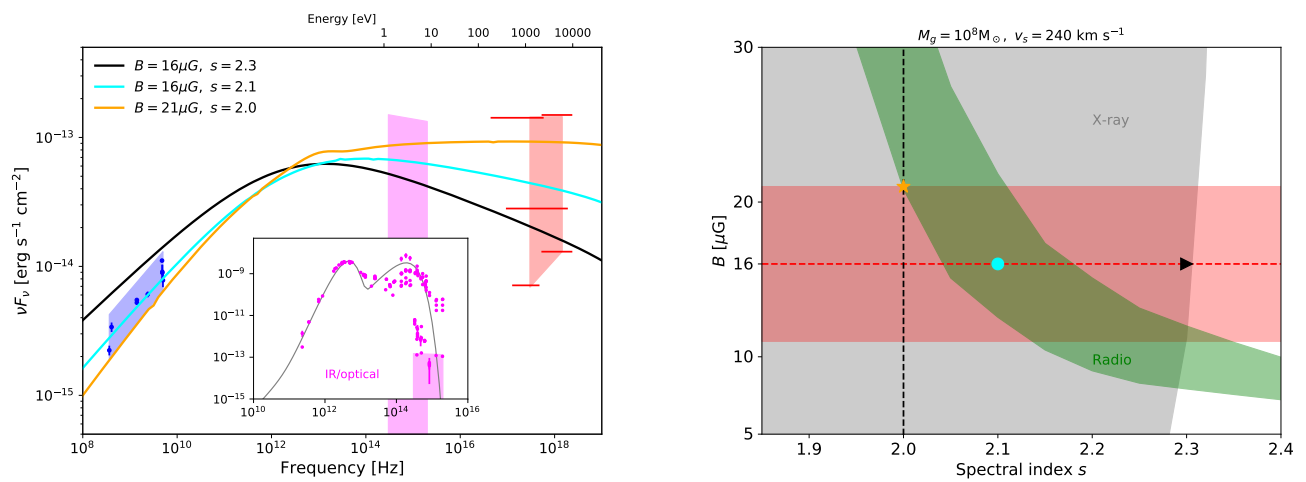

Figure 3: Left panel shows the spectral energy distribution for NGC 660, extending from the radio band to the $\mathrm{X}$-ray regime. In the right panel, the gray and green areas are $\mathrm{X}$-ray and radio constraints on $s-B$ plane. The red area shows the constraints on the magnetic from previous polarization studies, $16 \pm 5 \mu \mathrm{G}$.

the gas mass $M_{\mathrm{g}}$ and shock velocity $v_{s}$ for different magnetic fields. In each panel, blue and red areas correspond to the radio and $\mathrm{X}$-ray constraints and the black line shows the upper boundary under the UV constraint. The vertical dashed line and gray area show the constraints from the core region gas density $n_{\mathrm{g}} \lesssim 100 \mathrm{~cm}^{-3}$, whereas the horizontal dashed lines and gray area correspond to the strong shock requirements $(\mathscr{M} \simeq 10)$ for the temperature $10^{4} \mathrm{~K}$ and $10^{3} \mathrm{~K}$. The magenta dash-dotted contours correspond to different $p p$ optical depth $f_{p p, \mathrm{~g}}$. From Fig. 2, we find that a stronger magnetic field is favored to fit the radio, UV, and X-ray data simultaneously, since the spectral energy distribution of NGC 660 is consistent with a fast cooling synchrotron spectrum.

This simple single-zone model meets difficulty in explaining the radio and X-ray observations at the same time with a relatively lower $B$. This motivates us to exploit the chance of improving the fitting by varying the CR spectral index $s$. The constraints on the $B-s$ plane are shown in the right panel of Fig. 3. From this figure, we conclude that a moderately steeper CR spectrum with $2.1 \lesssim s \lesssim 2.3$ can alleviate the tension between the tensions between the radio and X-ray constraints. To show that our model's applicability can be extended to other similar systems, we also consider another well-studied galaxy formed through a merger, NGC 3256, as a supplementary template [17]. 


\section{Discussion and Conclusions}

In this paper, we present one multi-messenger scenario for the galaxy merging systems. We demonstrate that galaxy and cluster mergers can explain a significant portion of IceCube diffuse neutrino flux without violating the non-blazer EGB constraints. We also propose one simple onezone model to explain the radio and X-ray observations of the emitting region employing the secondary HE electron-positron pairs. It turns out that the multi-wavelength observations can be fitted simultaneously and this one-zone model is able to constrain the physical parameters in the merging region, e.g. $B, M_{\mathrm{g}}, v_{s}$.

One caveat is that in this simple one-zone model, the mass and velocity are strongly degenerated. Future neutrino and $\gamma$-ray observations would be helpful and indispensable to break such degeneracy since the spectral index can be unambiguously determined by the joint consideration of the neutrino and $\gamma$-ray spectra. Hence, we test the observability of galaxy mergers as a point source using the next-generation neutrino and gamma-ray detectors. From the left panel of Fig. 4, we find that the galaxy mergers can be identified as a point source with the IceCube-Gen2, if the source is well located, e.g. NGC 660. The horizontal lines in the right panel demonstrate the $\gamma$-ray fluxes from NGC 660 and NGC 3256 survived from $\gamma \gamma$ attenuations. From this figure we find that our model for the merging galaxies can be tested by $\mathrm{CTA}^{2}$. Above all, we conclude that the merging galaxies can be important candidates in the next generation multi-messenger studies and our model for the merging galaxies can be further tested by gamma-ray and neutrino observations with CTA and IceCube-Gen2.
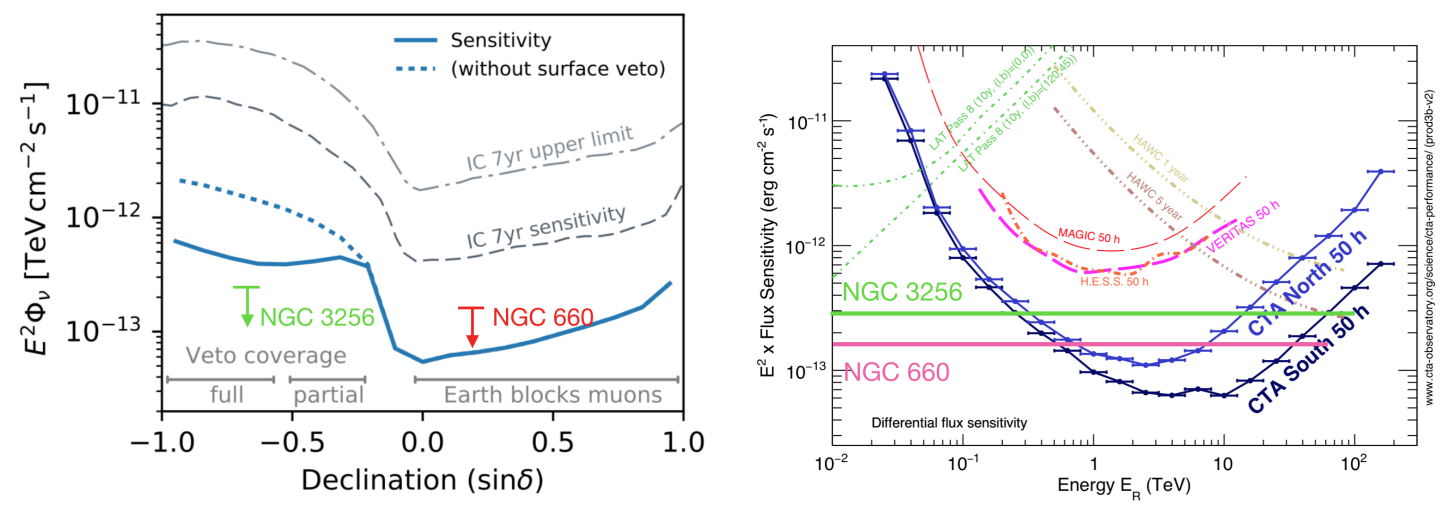

Figure 4: Detectability of NGC 660 and NGC 3256 as a point source of neutrinos and $\gamma$ rays. The IceCube-Gen2 sensitivity curves are taken from [18]

\section{References}

[1] M. G. Aartsen, M. Ackermann, J. Adams, J. A. Aguilar, M. Ahlers, M. Ahrens et al., Observation of High-Energy Astrophysical Neutrinos in Three Years of IceCube Data, Phys. Rev. Lett. 113 (2014) 101101 [1 405.5303$]$.

\footnotetext{
${ }^{2}$ CTA sensitivities are given by CTA Performance
} 
[2] M. G. Aartsen, K. Abraham, M. Ackermann, J. Adams, J. A. Aguilar, M. Ahlers et al., A Combined Maximum-likelihood Analysis of the High-energy Astrophysical Neutrino Flux Measured with IceCube, Astrophys. J. 809 (2015) 98 [1507.03991].

[3] M. G. Aartsen, K. Abraham, M. Ackermann, J. Adams, J. A. Aguilar, M. Ahlers et al., Observation and Characterization of a Cosmic Muon Neutrino Flux from the Northern Hemisphere Using Six Years of IceCube Data, Astrophys. J. 833 (2016) 3 [1607. 08006 ].

[4] M. Ahlers and F. Halzen, High-energy cosmic neutrino puzzle: a review, Reports on Progress in Physics 78 (2015) 126901.

[5] P. Mészáros, Astrophysical Sources of High-Energy Neutrinos in the IceCube Era, Annual Review of Nuclear and Particle Science 67 (2017) 45 [1708.03577].

[6] K. Kashiyama and P. Mészáros, Galaxy Mergers as a Source of Cosmic Rays, Neutrinos, and Gamma Rays, Astrophys. J. Lett. 790 (2014) L14 [1405 . 3262].

[7] B. Katz and E. Waxman, In which shell-type SNRs should we look for gamma-rays and neutrinos from P-P collisions?, JCAP 2008 (2008) 018 [0 706.3485 ].

[8] D. Caprioli and A. Spitkovsky, Simulations of Ion Acceleration at Non-relativistic Shocks. I. Acceleration Efficiency, Astrophys. J. 783 (2014) 91 [1310.2943].

[9] P. S. Behroozi, R. H. Wechsler and C. Conroy, The Average Star Formation Histories of Galaxies in Dark Matter Halos from z = 0-8, Astrophys. J. 770 (2013) 57 [1207. 610 5].

[10] M. T. Sargent, E. Daddi, M. Béthermin, H. Aussel, G. Magdis, H. S. Hwang et al., Regularity Underlying Complexity: A Redshift-independent Description of the Continuous Variation of Galaxy-scale Molecular Gas Properties in the Mass-star Formation Rate Plane, Astrophys. J. 793 (2014) 19 [1303.4392].

[11] C. Yuan, P. Mészáros, K. Murase and D. Jeong, Cumulative Neutrino and Gamma-Ray Backgrounds from Halo and Galaxy Mergers, Astrophys. J. 857 (2018) 50 [1712.09754].

[12] V. S. Berezinskii and A. I. Smirnov, Cosmic Neutrinos of Ultra High Energies and Detection Possibility, Astrophys Space Sci 32 (1975) 461.

[13] IceCube Collaboration, M. G. Aartsen, M. Ackermann, J. Adams, J. A. Aguilar, M. Ahlers et al., The IceCube Neutrino Observatory - Contributions to ICRC 2017 Part II: Properties of the Atmospheric and Astrophysical Neutrino Flux, arXiv e-prints (2017) arXiv:1710.01191 [1710.01191].

[14] M. Ackermann, M. Ajello, A. Albert, W. B. Atwood, L. Baldini, J. Ballet et al., The Spectrum of Isotropic Diffuse Gamma-Ray Emission between $100 \mathrm{MeV}$ and $820 \mathrm{GeV}$, Astrophys. J. 799 (2015) 86 [1410.3696].

[15] M. E. Filho, F. Fraternali, S. Markoff, N. M. Nagar, P. D. Barthel, L. C. Ho et al., Further clues to the nature of composite LINER/H II galaxies, A\&A 418 (2004) 429 [astro-ph / 0401593 ].

[16] R. T. Drzazga, K. T. Chyży, W. Jurusik and K. Wiórkiewicz, Magnetic field evolution in interacting galaxies, A\&A 533 (2011) A22 [1107.3280].

[17] C. Yuan, K. Murase and P. Mészáros, Secondary Radio and X-Ray Emissions from Galaxy Mergers, Astrophys. J. 878 (2019) 76 [1810.04155].

[18] IceCube-Gen2 Collaboration, :, M. G. Aartsen, M. Ackermann, J. Adams, J. A. Aguilar et al., The IceCube Neutrino Observatory - Contributions to ICRC 2017 Part VI: IceCube-Gen2, the Next Generation Neutrino Observatory, arXiv e-prints (2017) arXiv:1710.01207 [1710.01207]. 